\title{
A note from the editors: 2011 in numbers and developments foreseen for 2012
}

Eurosurveillance editorial team (eurosurveillance@ecdc.europa.eu) ${ }^{1}$

1. European Centre for Disease Prevention and Control (ECDC), Stockholm, Sweden

Citation style for this article:

Eurosurveillance editorial team. A note from the editors: 2011 in numbers and developments foreseen for 2012.

Euro Surveill. 2012;17(1):pii=20051. Available online: http://www.eurosurveillance.org/ViewArticle.aspx?Articleld=20051

Article published on 5 January 2012

Another year has passed by during which the public health community and Eurosurveillance faced both new and already familiar challenges. The toxin-producing Escherichia coli 0104 outbreak in Germany captured the attention of many public health experts over several months. West Nile virus became established in Greece after the outbreak in 2010, and public health experts were faced with the re-emergence of malaria in the same country.

Online and open access journals are increasing in numbers. Moreover, with 1.4 million scientific articles published annually, scientific journals are not the only outlet for scientific communication anymore. With more modern ways, the timeliness of communication and good quality of scientific publications become increasingly important. So do the additional functionalities and services offered by journals. Where does Eurosurveillance stand and where will it go?

In 2011, Eurosurveillance was the first scientific journal to cover the large outbreak of enteroaggregative Shiga toxin-producing $E$. coli $\mathrm{O}_{104}: \mathrm{H}_{4}$. We published a rapid communication on 26 May shortly after the outbreak had started. It was followed by a further 11 articles including an e-alert, an ad hoc scientific communication about an important event that should not wait until the next regular publication of Eurosurveillance. The high level of interest confirms the ability of Eurosurveillance to publish manuscripts about new developments of key interest to public health experts in a timely manner. As of 5 January 2012, the e-alert had been downloaded some 2,500 times and cited 19 times, according to the Scopus database.

The recent occurrence of cefixime-resistant gonorrhoea was covered in three rapid communications from Sweden, the United Kingdom and Austria. A report from Australia highlighted the first case of a Neisseria gonorrhoeae porA pseudogene false-negative PCR result caused by sequence variation and the first description of a clinical $N$. gonorrhoeae strain harbouring an N. meningitidis porA sequence.

Eurosurveillance was also the first scientific journal to report on outbreaks of HIV in injecting drug users in two European countries and on the risk for similar outbreaks in other European countries.

A comprehensive special issue on Chagas disease highlighted the relevance of this neglected tropical disease for Europe.

Overall, Eurosurveillance published in 2011 around 190 rapid communications and regular articles, as well as 16 editorials, 10 letters and 39 other items (such as

\section{FIGURE}

Time from submission to publication of articles in Eurosurveillance in 2011 ( $n=190)$
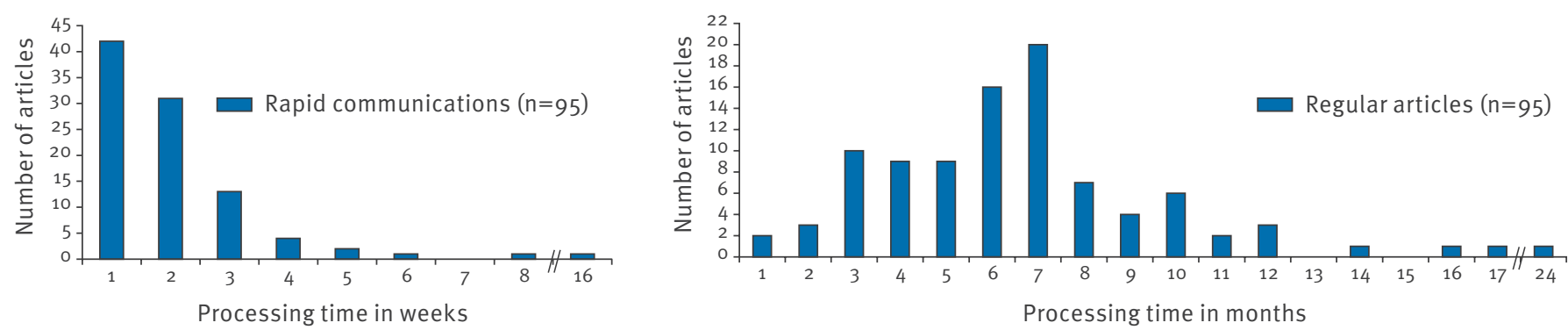
news and meeting reports). The average submissionto-publication time was 1.6 weeks for rapid communications and 28 weeks for regular papers. Some $77 \%$ $(n=73)$ of the rapid communications were published within two weeks from submission, and $73 \%(n=69)$ of the regular articles were published within seven months (Figure). The rejection rate has remained at 43\% in 2011 for rapid communications and increased to $75 \%$ for regular papers (63\% in 2010). Submissions came from more than 37 countries and covered mostly EU countries as well as 12 countries not represented on the Eurosurveillance board.

A milestone for Eurosurveillance was a scientific seminar organised for the occasion of the 15-year anniversary celebration on 8 November 2011.

In 2011, as in previous years, the team has been supported by numerous experts from across the globe. Some had a formal role as board members, authors and peer reviewers; others played a more informal role providing ad hoc topical input and advice. In 2011, some 380 reviewers supported us and their names are published in this issue. We are grateful for their advice as well as for the informal input from our network of supporters.

The year 2012 has just started. Much will remain the same. We will keep our focus, which is on the surveillance, prevention and control of communicable diseases in Europe and remain vigilant for new developments in this area and related fields.

But there will also be some new developments. We will expand our editorial board and welcome Heath Kelly, Head of Epidemiology Unit, Victorian Infectious Diseases Reference Laboratory in Victoria, Australia as new associate editor.

We plan to improve the efficiency and transparency of our workflows by implementing an online submission system. Acknowledging the importance of social media, we will set up a twitter account in this month and hope that many of you will follow us.

In mid-2012, we will receive our first official impact factor. It will complement the acknowledged capacity of the journal to impact on public health through providing the public health community with information necessary for the implementation of measures to prevent and control communicable diseases.

Our main aim in 2012 remains to serve our audience and to balance timeliness, public health relevance, quality and scientific content for the benefit of all those concerned with surveillance, prevention and control of communicable disease in Europe and beyond. We count on the good collaboration with our board, authors, reviewers and readers to achieve this aim. 